\title{
THE CONVEX HULL PROPERTY OF NONCOMPACT HYPERSURFACES WITH POSITIVE CURVATURE
}

\author{
STEPHANIE ALEXANDER AND MOHAMMAD GHOMI
}

\begin{abstract}
We prove that in Euclidean space $\mathbf{R}^{n+1}$, every metrically complete, positively curved immersed hypersurface $M$, with compact boundary $\partial M$, lies outside the convex hull of $\partial M$ provided that $\partial M$ is embedded on the boundary of a convex body and $n>2$. For $n=2$, on the other hand, we construct examples which contradict this property.
\end{abstract}

\section{INTRODUCTION}

As we showed recently [2], a compact, immersed positively curved hypersurface of Euclidean space satisfies a Convex Hull Property (CHP), dual to the classical one for nonpositively curved hypersurfaces [13]. Our CHP states that the hypersurface lies outside the convex hull of its boundary, provided the boundary satisfies certain required conditions, e.g., it is embedded on the boundary of a convex body. A proof of a version of this fact has also been obtained by Guan and Spruck [9] via their work on Monge-Ampére equations. In this note we study generalization of CHP to noncompact, (metrically) complete hypersurfaces, and uncover a surprising dichotomy: the noncompact version of CHP fails in $\mathbf{R}^{3}$ but holds in higher dimensions.

Theorem 1.1. Let $M$ be a connected, smooth $n$-manifold with compact boundary, $n \geq 3$. Let $f: M \rightarrow \mathbf{R}^{n+1}$ be a complete smooth immersion with positive sectional curvature, and $C:=\operatorname{conv} f(\partial M)$ be the convex hull of the image of the boundary of $M$ (we assume $\partial M \neq \emptyset$ ). Suppose that $f(\partial M) \subset \partial C$, and $f$ is an embedding on each component of $\partial M$. Then the image of the interior of $M$ lies completely outside the convex hull of the image of its boundary,

$$
f(\operatorname{int} M) \cap C=\emptyset .
$$

Further, $f$ is an embedding on $\partial M$, and $M$ is homeomorphic to the complement of a finite point set in the closure of a component of $\partial C-f(\partial M)$ with boundary $f(\partial M)$. (If $C$ is degenerate, i.e. $\operatorname{int} C=\emptyset$, then we take $\partial C$ to be two copies of $C$ glued along their relative boundaries.)

If $M$ is compact, this theorem is proved for $n \geq 2$ in [2]. (In this case, there is no deleted point set.) Below we give an example (Figure 1) showing that when $M$ is noncompact and $n=2$, the theorem fails.

1991 Mathematics Subject Classification. Primary 53A07, 53C45; Secondary 53C21, 53C23.

Key words and phrases. Convex hull, positive curvature,

The second author was partially supported by NSF Grant DMS-0204190, and CAREER award DMS-0332333. 
In [2], CHP theorems are proved for other classes of hypersurfaces as well, including nonnegatively curved hypersurfaces, and nonsmooth, locally convex hypersurfaces (see Theorem 2.2 below). The proof of Theorem 1.1 depends on this work and earlier work of Robert Currier and the first author concerning hypersurfaces that are nonnegatively curved off compact sets (see Theorem 2.1 below). A version of the CHP theorems had been originally conjectured in the second author's Ph.D. thesis [6, Prob. E.0.5], [7].

\section{Preliminaries}

We say a smooth immersion $f: M \rightarrow \mathbf{R}^{n+1}$ is complete if $M$ is a complete metric space with respect to the intrinsic metric induced by $f$. Thus, in our terminology, a complete hypersurface may have boundary (it need not be geodesically complete). It is still true that the compact subsets of $M$ are the closed and (intrinsically) bounded ones. (See [4] for Cohn-Vossen's extension of the Hopf-Rinow theorem.)

By an end representative in $M$, we mean an unbounded component of the complement of a compact subset of $M$. An end is an equivalence class of nested decreasing sequences of end representatives that eventually lie outside every compact subset of $M$. Two sequences are equivalent if any member of either contains a truncation of the other. By a convex body in $\mathbf{R}^{n+1}$, we mean a convex set with interior points (our convex bodies need not be compact). A convex cap is the intersection of the boundary of a convex body with an open halfspace.

Theorem 2.1. Let $M$ be a connected, smooth $n$-manifold with compact boundary, $n \geq 3$. Let $f: M \rightarrow \mathbf{R}^{n+1}$ be a complete smooth immersion with positive sectional curvature. Then each end of $M$ has a convex representative, that is, one that is embedded onto a convex cap.

Proof. In [1], the same conclusion is obtained for a complete hypersurface without boundary having positive sectional curvature off a compact subset. The proof applies almost verbatim to the present case. Indeed, it may be simplified because [1] also discusses the case with sectional curvature nonnegative and nullity of the second fundamental form at most 1 (off a compact subset). Thus we present only an outline of the arguments, and refer the reader to [1] for more details.

The main idea for the proof is as follows. Let $C$ be a subset of $M$ which is embedded by $f$ onto a convex cap. Note that $C$ is an end representative if and only if (i) the closure of $C$ is noncompact, but (ii) $\partial C$ is compact. We need to show that each end of $M$ has such a representative. This is achieved by adapting the method of slicing with moving hyperplanes first used by van Heijenoort [11] (see [1, Thm. 2]).

Suppose $H \subset \mathbf{R}^{n+1}$ is a hyperplane which does not intersect $f(\partial M)$. Then by a transversality argument, each component $N$ of $f^{-1}(H)$ which is not a singleton is an $(n-1)$-dimensional submanifold without boundary of $M$, on which $f$ acts as a complete, positively curved hypersurface immersion into $H$. Since $n \geq 3$, it follows then from a classical theorem of Stoker [14], that $f$ embeds $N$ onto the boundary of a convex subset of $H$ (here is where we use the dimension restriction). 
The above observation is used to show that for any point $p \in M$ such that $f(p)$ lies outside a Euclidean ball containing $f(\partial M)$, slicing by rotated hyperplanes through $f(p)$ produces a subset $C_{p}$ of $M$ that is embedded onto a convex cap, and has boundary containing $p$ and intersecting $\partial M$ (see [1, Lemma 2]).

Since $M$ has positive curvature, an argument of Okayasu [12] implies that $f(M)$ is unbounded. In particular, there exists a sequence of points $p_{i}$ of $M$ such $f\left(p_{i}\right)$ lies outside a ball containing $f(\partial M)$ and is unbounded. The existence of convex caps $C_{p_{i}}$ together with compactness of $\partial M$, may then be used to show that in each end there is a subset that is embedded onto a convex cap $X$, and has noncompact closure (see [1, Lemma 3]). If this subset has compact boundary, then we are done; otherwise, we proceed as follows.

Let $v$ be a direction in the "recession cone" of conv $X$, and $X_{v}$ be the union of slices of $M$ by hyperplanes $\langle y, v\rangle=t$ for $t$ sufficiently large. If these slices are compact, $X_{v}$ is the desired end representative. If not, we may choose $w$ in the common recession cone of these slices, and find an end representative of the form $X_{v} \cup X_{w}$. Then a convexity argument identifies a family of parallel hyperplanes such that the corresponding slices of $X_{v} \cup X_{w}$ are compact and form the desired end representative.

Now suppose $f: M \rightarrow \mathbf{R}^{n+1}$ is an immersion of a topological $n$-manifold (i.e., a locally one-to-one continuous map). We say $f$ is locally convex if $f$ has an extension $\widetilde{f}$ to a manifold without boundary $\widetilde{M}$, where every point $p \in M$ has a neighborhood $U_{p}$ in $\widetilde{M}$ that is embedded by $\widetilde{f}$ into the boundary of a convex body $K_{p} \subset \mathbf{R}^{n+1}$. (The assumption that $f$ extends to a collaring manifold $\widetilde{M}$ is a compatibility condition on the $K_{p}$ for boundary points $p$. Without the compatibility condition, the CHP need not hold; see [2] for examples and further discussion.) If, for all $p \in \partial M, U_{p}$ may be chosen so that $f\left(U_{p} \cap M\right)$ contains no line segments, we say $f$ is locally strictly convex on a neighborhood of $\partial M$.

We need the following CHP theorem for locally convex hypersurfaces which are compact:

Theorem $2.2([2])$. Let $M$ be a compact connected topological $n$-manifold, $n \geq 2$, $f: M \rightarrow \mathbf{R}^{n+1}$ be a locally convex immersion, and $C:=\operatorname{conv} f(\partial M) \neq \emptyset$. Suppose that $f(\partial M) \subset \partial C, f$ is locally strictly convex on a neighborhood in $M$ of $\partial M$, and $f$ is an embedding on each component of $\partial M$. Then

$$
f(\operatorname{int} M) \cap C=\emptyset .
$$

Further, $f$ is an embedding on $\partial M$, and $M$ is homeomorphic to the closure of a component of $\partial C-f(\partial M)$ with boundary $f(\partial M)$.

\section{Proof of Theorem 1.1}

We proceed in two parts: first we observe that $M$ has only finitely many ends, by applying a theorem of Greene and $\mathrm{Wu}[10]$, and then we use Theorem 2.1 to "clip off" the ends, and reduce the proof to that of Theorem 2.2. 
I. Since $\partial M$ is compact, it has only finitely many components $\partial M_{i}$, and each $\partial M_{i}$ is a closed $(n-2)$-dimensional manifold. Thus, since $f$ is an embedding on $\partial M_{i}$, $f\left(\partial M_{i}\right)$ is an embedded $(n-2)$-dimensional closed submanifold of $\partial C$. But, since $\partial M$ is compact, $C$ is a compact convex set. In particular, if $\operatorname{int}(C) \neq \emptyset$, then $\partial C$ is homeomorphic to the sphere $\mathbf{S}^{n}$. So, by the generalized Jordan-Brouwer separation theorem [3], $f\left(\partial M_{i}\right)$ is the boundary of a compact connected manifold $X_{i} \subset \partial C$. If $\operatorname{int} C=\emptyset$, then $\partial M_{i}$ lies in a hyperplane. In this case, again by the Jordan-Brouwer theorem, $\partial M_{i}$ bounds a compact connected manifold $X_{i}$.

Gluing each $X_{i}$ to $M$ along $\partial M_{i}$, we obtain a manifold without boundary $M^{*}$. Let $U_{i}$ be an open neighborhood of $X_{i}$ with compact closure in $M^{*}$. In the overlapping regions $U_{i} \cap M$, we may use a partition of unity to blend in the metrics in the standard way, to obtain a complete smooth Riemannian manifold $M^{*}$ which is positively curved off a compact set. As Greene and Wu showed [10], building on the method of Cheeger and Gromoll [5], $M^{*}$ is diffeomorphic to the interior of a compact manifold with boundary. In particular, $M^{*}$ has only finitely many ends. But each end of $M$ is also an end of $M^{*}$. Thus $M$ has only finitely many ends.

II. By Theorem 2.1, each end of $M$ has a convex representative $E_{i}$. So there are a convex body $B_{i}$ and a hyperplane $H_{i}$, such that $f$ embeds $E_{i}$ onto the intersection of $\partial B_{i}$ with the open halfspace $H_{i}^{+}$. Since $C$ is compact, we may move $H_{i}$ parallel to itself inside $H_{i}^{+}$until $H_{i}^{+} \cap C=\emptyset$. Replacing $E_{i}$ by the corresponding (smaller) convex representative, we may then assume that

$$
f\left(E_{i}\right) \cap C=\emptyset .
$$

Now we "clip off the ends" as follows. For each $E_{i}$, let $D_{i}:=H_{i} \cap B_{i}$ be the corresponding convex body in $H_{i}$ and set $D:=\cup_{i} D_{i}$. Further let $M^{\prime}:=M-\cup_{i} E_{i}$. By part I, $D$ and $M^{\prime}$ are both compact manifolds with boundary, and $\partial D=\partial M^{\prime}-$ $\partial M$. So, gluing $D$ to $M^{\prime}$, by gluing each $D_{i}$ to $\partial E_{i}$, we obtain a compact manifold $\bar{M}$ with $\bar{M}-\cup D_{i}=M-\cup E_{i}$. In particular $\partial M=\partial \bar{M}$, since, by construction, $H_{i} \cap C=\emptyset$.

Also we modify $f$ accordingly by setting it equal to the identity on each $D_{i}$ to obtain a (nonsmooth) immersion $\bar{f}: \bar{M} \rightarrow \mathbf{R}^{n+1}$. Since $f$ is locally convex (see $[2$, Lemma 3.1]), $\bar{f}$ will be locally convex as well.

Thus we obtain a compact manifold $\bar{M}$, and a locally convex immersion $\bar{f}: \bar{M} \rightarrow$ $\mathbf{R}^{n+1}$ which satisfy the hypotheses of Theorem 2.2. So $f(\operatorname{int} \bar{M}) \cap C=\emptyset$. Since (int $\left.M-\cup_{i} E_{i}\right) \subset \operatorname{int}(\bar{M})$, this yields

$$
f\left(\operatorname{int} M-\cup_{i} E_{i}\right) \cap C=\emptyset .
$$

Together, (3.1) and (3.2) complete the proof of CHP.

To obtain the topological conclusions of Theorem 1.1, note that, by Theorem 2.2, $\bar{M}$ is homeomorphic to the closure of a component of $\partial C-f(\partial \bar{M})$ bounded by $f(\partial \bar{M})$. Since $\partial \bar{M}=\partial M$, it remains then to show that $M$ is homeomorphic to $\bar{M}$ minus a finite number of points. This follows because each $E_{i}$ is homeomorphic to $\partial B_{i} \cap H_{i}^{+} \simeq \mathbf{S}^{n-1} \times \mathbf{R}$, which is the same as an $n$-disk minus a point, e.g., $D_{i}$ minus a point. 
Note 3.1. If $n=2$, Theorem 1.1 still holds for embeddings because Theorem 2.1 does.

\section{EXAMPLE}

Here we construct an immersed surface in $\mathbf{R}^{3}$ that satisfies the hypotheses of Theorem 1.1 but enters the convex hull of its boundary. We start with a compact surface which we then transform to a complete one by a projective transformation.

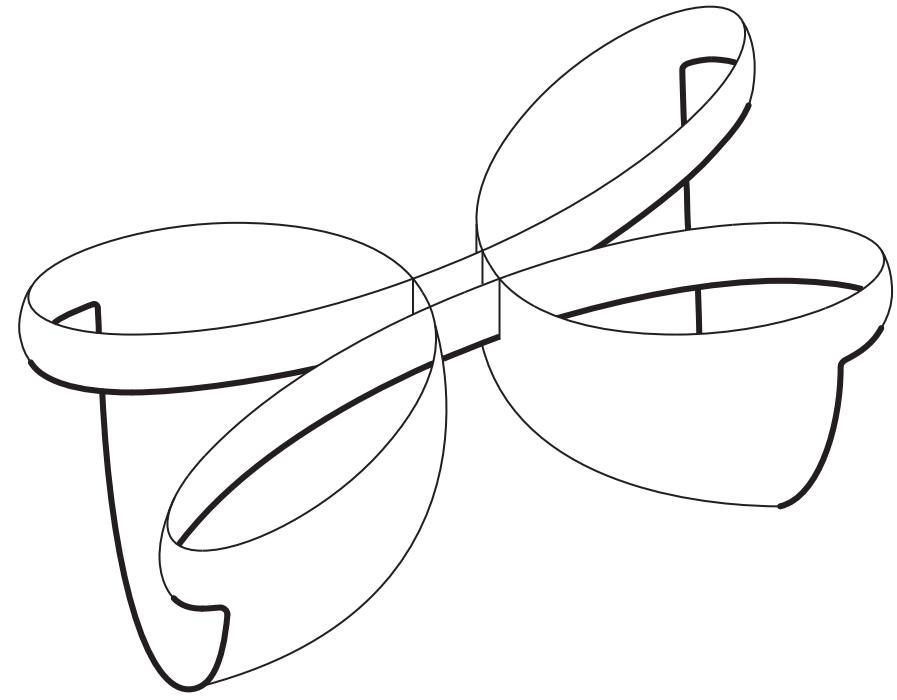

Figure 1

Consider the immersed surface $M$ illustrated in Figure 1. It is composed of a pair of quarter spheres, which are attached by a pair of positively curved ribbons. The technique for constructing such ribbons has been well studied and formalized by Gluck and Pan [8]. Note that $M$ has an embedded boundary component, $\Gamma_{1}$ (the darker curve), which lies on the boundary of its own convex hull $C$. Further, int $M \cap C \neq \emptyset$. In particular most of each of the quarter spheres lies in $C$. The other boundary component of $M, \Gamma_{2}$, is an immersed planar curve in the shape of a clover leaf.

Suppose that $\Gamma_{2}$ lies in the $x y$-plane and consider the projective transformation $T$ given by

$$
(x, y, z) \stackrel{T}{\longmapsto}(x / z, y / z, 1 / z) .
$$

This transformation maps $M-\Gamma_{2}$ to a complete hypersurface bounded by a single curve $T\left(\Gamma_{1}\right)$. Since projective transformations preserve the sign of curvature, $T(M)$ has positive curvature. Further, $T$ preserves line segments, and therefore sends convex bodies to convex bodies. So $T\left(\Gamma_{1}\right)$ again lies on the boundary of its own convex hull, because $T\left(\Gamma_{1}\right) \subset T(\partial C)=\partial T(C)=\partial \operatorname{conv} T\left(\Gamma_{1}\right)$. Finally, since $\operatorname{int}(M) \cap C \neq \emptyset$, it follows that

$$
\operatorname{int} T(M) \cap T(C)=T(\operatorname{int} M) \cap T(C)=T(\operatorname{int} M \cap C) \neq \emptyset .
$$


Hence $T(M)$ is the desired surface.

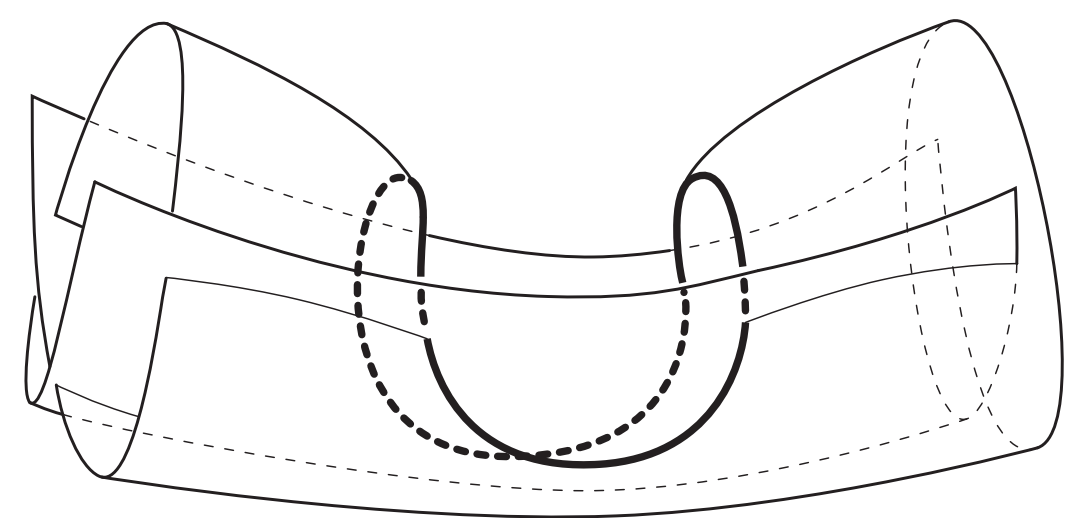

FiguRE 2

Another counterexample to the noncompact CHP for $\mathrm{n}=2$, which might be easier to visualize, is depicted in Figure 2. This surface has the same topology as the previous example, but has a somewhat more complicated end, as it intersects itself 6 times (the end of the previous example has 4 symmetrically placed self-intersections).

\section{REFERENCES}

[1] S. Alexander and R. Currier, Nonnegatively curved ends of Euclidean hypersurfaces, Geometriae Dedicata 40 (1991), 29-43.

[2] S. Alexander and M. Ghomi, The convex hull property and topology of hypersurfaces with nonnegative curvature, Adv. Math., 180 (2003), 324-354 .

[3] G. Bredon, Topology and Geometry, Springer-Verlag, New York, 1993.

[4] H. Busemann, Recent Synthetic Differential Geometry, Springer-Verlag, New York, 1970.

[5] J. Cheeger and D. Gromoll, On the structure of complete manifolds of nonnegative curvature, Ann. of Math. 96 (1972), 413-443.

[6] M. Ghomi, Strictly convex submanifolds and hypersurfaces of positive curvature, Ph.D. Thesis, Johns Hopkins University, 1998.

[7] — Strictly convex submanifolds and hypersurfaces of positive curvature, J. Differential Geom. 57 (2001), 239-271.

[8] H. Gluck and L. Pan, Embedding and knotting of positive curvature surfaces in 3-space, Topology 37 (1998), no. 4, 851-873.

[9] B. Guan and J. Spruck, The existence of hypersurfaces of constant Gauss curvature with prescribed boundary, 62 (2002), 259-287 .

[10] R. Greene and H. Wu. $C^{\infty}$ convex functions and manifolds of positive curvature, Acta Math. 137 (1976), 209-245.

[11] J. van Heijenoort, On locally convex manifolds, Comm. Pure Appl. Math., 5(1952), 223-242.

[12] T. Okasayu, Some results in geometry of hypersurfaces, Kodai Math. J., 9 (1986), 77-83.

[13] R. Osserman, The convex hull property of immersed manifolds, J. Differential Geom., 6 (1971/72), 267-270.

[14] J. Stoker, Über die Gestalt der positiv gekrümm ten offenen Flächen im dreidimensionalen Raume, Compositio Math. 3 (1936), 55-89. 
Department of Mathematics, University of Illinois,Urbana, IL 61801

E-mail address: sba@math.uiuc.edu

$U R L$ : www. math.uiuc.edu/ sba

School of Mathematics, Georgia Institute of Technology, Atlanta, GA 30332

E-mail address: ghomi@math.gatech.edu

$U R L$ : www . math. gatech.edu/ ghomi

Current address: Dept. of Mathematics, Penn State University, State College, PA 16803 